\title{
MIRON: MIPv6 Route Optimization for NEMO
}

\author{
Carlos Jesús Bernardos, Marcelo Bagnulo, María Calderón \\ Universidad Carlos III de Madrid - Dep. Ingeniería Telemática \\ Av. Universidad, 30; 28911 Leganés, Madrid - España \\ Telephone: +34916248859 Fax: +34916248749 \\ E-mail: \{cjbc, marcelo, maria\}@it.uc3m.es
}

\begin{abstract}
Network Mobility (NEMO) Basic Support Protocol enables mobile networks to change their point of attachment to the Internet, while preserving established sessions of the nodes within the mobile network. NEMO basic solution is an extension of Mobile IPv6 and it is based on tunnelling, which leads to suboptimal routing, packet overhead and latency, especially when nesting (i.e. a mobile network that contains other mobile networks within) is involved. In this article we present a route optimisation solution for mobile networks based on Mobile IPv6. The goal is to use the route optimisation support for Mobile IPv6 available in the Correspondent Nodes to provide route optimisation for mobile networks. The solution also supports nested mobile networks without requiring additional tunnelling, thus reducing packet overhead and latency with regard to Network Mobility basic solution.
\end{abstract}

\section{INTRODUCTION}

As the demand for a ubiquitous Internet increases, network access has to be provided in more and more heterogeneous environments. In particular, the demand for Internet access in mobile platforms such as trains, buses and ships is constantly increasing. In order to satisfy such demands, the technical community is working on the design of the required protocols to provide what has been called Network Mobility support. In particular, a working group called NEMO has been created within the IETF to extend the basic end-host mobility support protocol, MIP [1] [2], to provide mobile network support.

In more precise terms, a Network that Moves (NEMO) a mobile network - can be defined as a network whose attachment point to the Internet varies with time. The router within the NEMO that connects to the Internet is called the Mobile Router (MR). It is assumed that the NEMO has a Home Network where it resides when it is not moving. Since the NEMO is part of the Home Network, the Mobile Network

The work described in this paper is based on results of IST FP6 Integrated Project DAIDALOS [16]. DAIDALOS receives research funding from the European Community's Sixth Framework Programme. Apart from this, the European Commission has no responsibility for the content of this paper. This paper may contain forward-looking statements relating to advanced information and communication technologies. Neither the DAIDALOS project consortium nor the European Community does accept any responsibility or liability for any use made of the information provided in this paper. has configured addresses belonging to the address block assigned to the Home Network (Home Prefix). These addresses remain assigned to the NEMO when it is away from home. Naturally, these addresses only have topological meaning when the NEMO is at home. When the NEMO is away from home, packets to the Mobile Network Nodes (MNNs) will still be routed to the Home Network. Additionally, when the NEMO is away from home, i.e. it is in a visited network, the mobile router acquires an address from the visited network, called the Care-of Address (CoA), where the routing architecture can deliver packets without additional mechanisms.

The goal of the network mobility support mechanisms is to preserve established communications between the MNN and external Correspondent Nodes (CNs) through movement. Packets of such communications will be addressed to the MNN' addresses, that belong to the Home Prefix, so additional mechanisms to forward packets between the Home Network and the NEMO are needed. As the informed reader may notice, such mechanisms are somehow similar to the ones required to provide end node mobility support, so the idea is to extend node mobility support to cope with Network Mobility. However, the network mobility case presents some specific issues that have to be addressed properly. For instance, a network mobility solution has to cope with nested NEMOs, i.e. a NEMO that contains another NEMO within. This scenario is more common that it may seem at first sight. For instance, consider the case of a NEMO build in a train to provide Internet access to travellers. Now, consider a user with a Personal Area Network formed by a traveller's handheld devices, such the PDA and/or the mobile phone, and its laptop. In this case, the mobile network of the train is the parent NEMO that receives a sub-NEMO that is the PAN. The solution to support NEMOs has to provide proper support for this scenario.

The basic solution for network mobility support [3] essentially creates a bi-directional tunnel between a node located in the Home Network of the NEMO, the Home Agent (HA), and the CoA of the MR (Fig. 1). This solution is similar to the solution proposed for host mobility support, MIPv6 [2], without including the route optimisation support. Actually, the protocol is similar and the existing Binding Update (BU) message is extended to inform the HA about the IP address (i.e. CoA of the MR) of the NEMO's side of the 


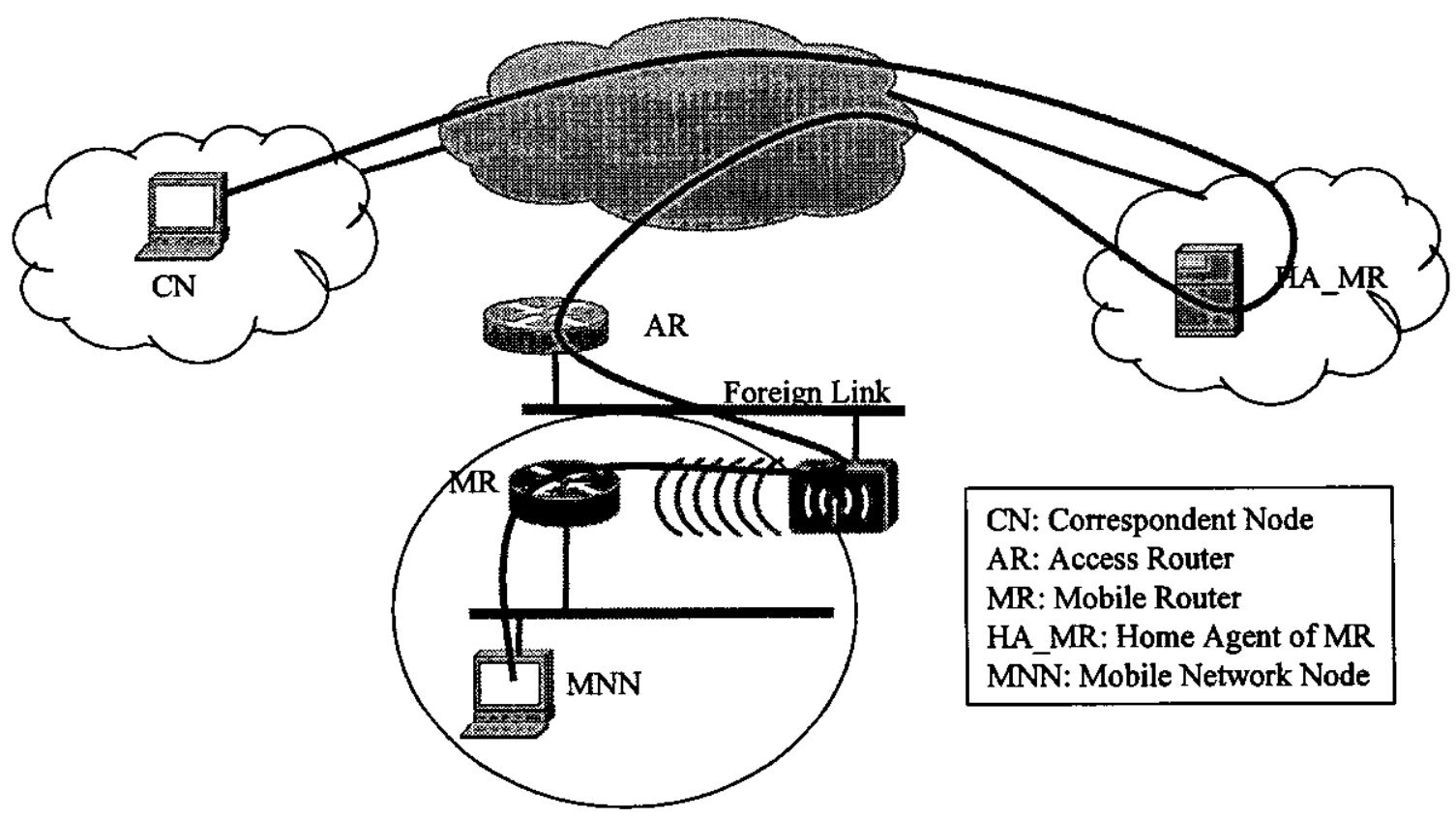

Fig. 1. NEMO Basic Support Protocol operation

tunnel through which the HA has to forward the packets addressed to the Home Prefix. The main limitation of this basic solution is that it forces triangular routing i.e. packets are always forwarded through the Home Agent following a suboptimal path. This problem is exacerbated when considering nested mobility, since in this case the packets are forwarded through all the Home Agents of all the mobile networks involved. This is because each sub NEMO obtains a CoA that belongs to the Home Prefix of its parent NEMO. Such $\mathrm{CoA}$ is not topologically meaningful in the current location, since the parent NEMO is also away from home, and packets addressed to the CoA are forwarded through the HA of the parent NEMO, as it is illustrated in Fig. 2. This behaviour of the basic solution not only causes suboptimal routing but it also introduces extra overhead, since each Home Agent tunnels the packet to the correspondent mobile router. This means that multiple IP headers will be inserted in the original packet, reducing MTU and increasing overhead.

Because of all the limitations identified above, it seems desirable to provide some route optimisation support that allows direct packet exchange between the $\mathrm{CN}$ and the $\mathrm{MNN}$ without passing through any $\mathrm{HA}$ and without inserting extra IP headers. In MIPv6, route optimisation is achieved when the Mobile Node (MN) sends a Binding Update (BU) message to the $\mathrm{CN}$ informing about the CoA address where its permanent address, which belongs to the Home Network i.e. Home Address (HoA) - is currently reachable. Because of security issues, the CN must verify that the CoA included in the BU message corresponds to the claimed HoA. Such verification is achieved through the Return Routability (RR) procedure, in which the $\mathrm{CN}$ informs the $\mathrm{MN}$ about two different secrets, one of which is sent to the HoA and the other one is sent to the MN's CoA. So, when the MN sends a BU it also includes in the message both secrets, proving that it is able to receive packets addressed to its $\mathrm{HoA}$ and current CoA.

This paper proposes a route optimisation solution for NEMOs based on MIPv6. The goal is to use the route optimization support for MIPv6 available in the CN to provide route optimization for NEMOs. Such solution would profit from the installed base of CNs that currently support MIPv6 route optimisation, so its adoption would be easier than if new CN mechanisms were required. The rest of the paper is structured as follows: in section II the proposed solution (MIRON) is presented in detail, both for the case of a single NEMO and its extension to support nested mobile networks. In section III, an overview of related work is presented, describing other proposals of route optimisation in the NEMO context. In section IV, a performance evaluation of MIRON, in comparison with NEMO basic support and a route optimisation proposal, named Reverse Routing Header, is presented. In section V, some implementation notes are provided. Finally, section VI is devoted to conclusions and future work. 


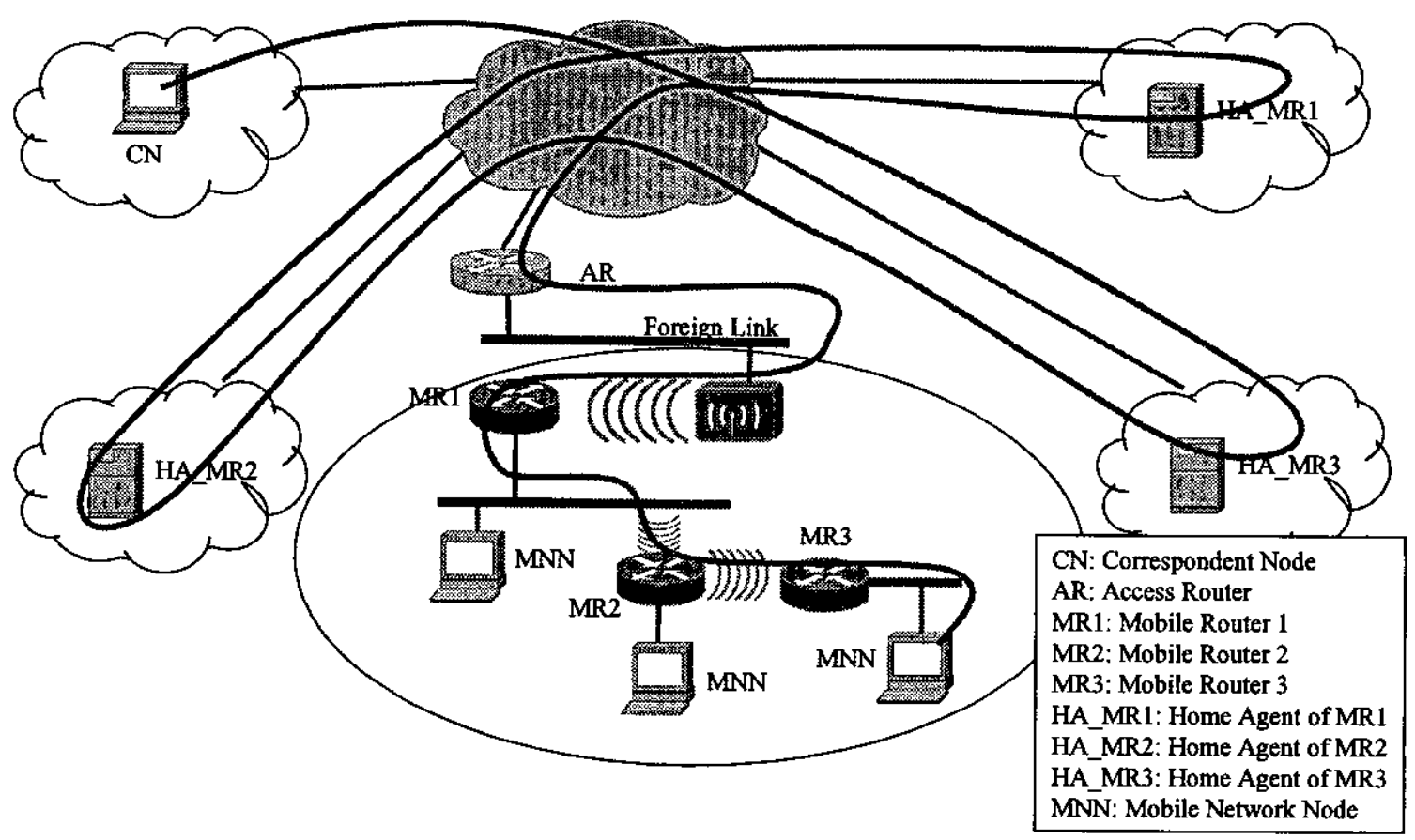

Fig. 2. Operation of NEMO Basic Support with nested mobile networks

\section{MIPv6 ROUTE OPTIMEZATION FOR NEMO (MIRON)}

We will present now a proposal for a Route Optimization mechanism for Local Fixed Nodes (i.e. MNN without MIP support) located into a NEMO. First, we will describe the goals of the solution and, finally, we will detail the solution both for a single NEMO and the extension needed for supporting nested NEMOs.

\section{A. Single Mobile Network}

\section{A.1. Goals and requirements}

This proposal provides route optimization between a Local Fixed Node (LFN) and a CN outside the NEMO, routing packets directly between the $\mathrm{MR}$ and the $\mathrm{CN}$, and avoiding the tunnel between the MR and the MR's HA.

One of the requirements for the NEMO support, as stated in [5], is that the proposed solutions have to provide transparent mobility support for LFNs. So, in order to be consistent with the requirements imposed to the NEMO Basic Solution, transparent support for route optimization is one of the design goals for the proposed solution.

A key issue to simplify the adoption of the proposed solution is to preserve the $\mathrm{CN}$ operation unchanged, i.e. the solution does not require additional mechanisms on the $\mathrm{CN}$. Changing the operation of $\mathrm{CN}$ would hinder the deployability of the solution, since it implies modifying every node in the Internet (every node is a potential CN). Nevertheless, it is assumed that the $\mathrm{CN}$ supports the Route Optimization mechanism required for a CN in MIPv6.

The mechanism here proposed basically consists in performing Mobile IPv6 Route Optimization procedure [1] between the MR (on behalf of the LFN) and the CN. In this way, latency is reduced since triangular routing through the HA is avoided and also the overhead introduced by the NEMO basic solution is diminished, especially in nested environments. The optimization proposed here is performed by the sole MRs and HAs, requiring some changes in them from the operation defined in [3].

\section{A.2. Proposed mechanism}

Basically, the idea is to enable a MR to behave as a proxy for the LFN, by performing the packet exchange required by the MIPv6 Route Optimization procedure. In order to do that, when the MR identifies a traffic flow between a $\mathrm{CN}$ outside the NEMO and a LFN inside the NEMO, it starts the procedure described in [4] in order to perform Route Optimization with the involved CN. The MIPv6 Route Optimization process is achieved when the MN sends the Binding Update message (BU) to the $\mathrm{CN}$. In this particular case the required $B U$ message sent by the MR to the $C N$ will contain the MR's CoA as the MN's CoA (since MR's CoA is the only topologically meaningful address available to the NEMO) and the LFN's address as the MN's HoA. However, MIPv6 requires the performance of the Return Routability procedure in order to validate the exchange of binding messages. 


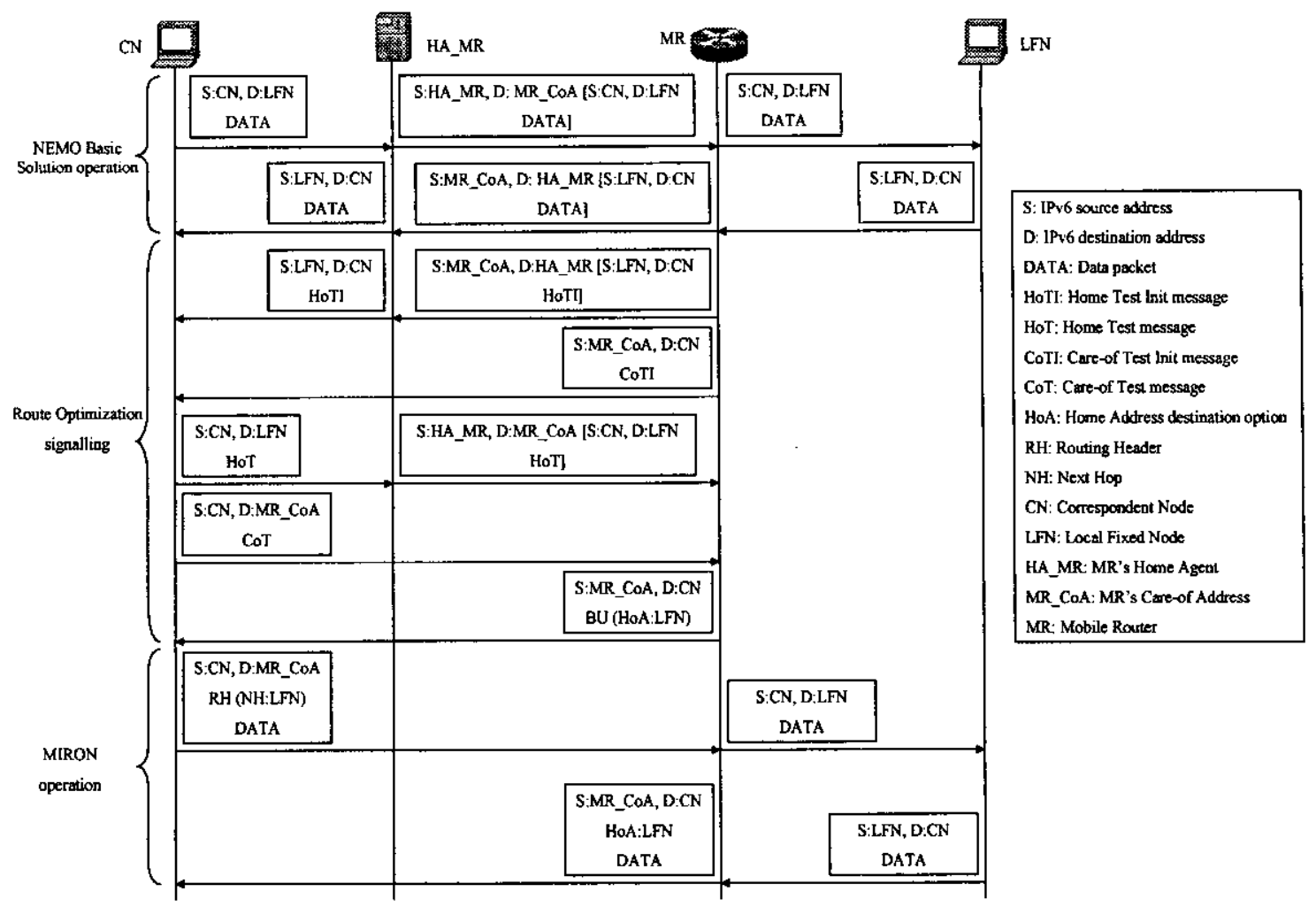

Fig. 3. Operation of the proposed mechanism

Therefore, the MR has to perform the Mobile IPv6 Return Routability procedure [4] on behalf of the LFN. Such procedure involves sending Home Test Init (HoTI) and Careof Test Init (CoTT) messages to the CN and the processing of the replies (Home Test message - HoT - and Care-of Test message - CoT). The HoTI message should be sent with source address set to the LFN's address through the MR's HA (HA MR). The CoTI message should be sent with source address set to the MR's CoA. After the processing of the HoT and CoT messages, the MR can now send the BU message to the CN on behalf of the LFN. This packet has to carry the MR's CoA as source address (acting as the MN's COA) and has to carry a Home Address destination option set to the LFN's address (acting as the MN's HoA).

The HoTI and HoT messages of the RR procedure should be protected by IPSec, as stated in [1], in order to avoid some potential security attacks. In this proposal, these messages are protected by IPSec, using the Security Association (SA) defined by the NEMO Basic Solution [3] between the MR and its own HA.

After this procedure has been completed, packets of the CN-LFN communication will experience MIPv6 Route optimization. Therefore, packets from the CN to the LFN will carry the MR's CoA as destination address, and will also carry a Type 2 Routing Header with the LFN's address as next hop. The MR should process the packet, eliminating the Routing Header, recalculating checksums (if needed) and delivering the packet to the LFN as a normal IPv6 packet. In the other sense, the MR receives packets from the LFN addressed to the CN carrying the LFN's address as IPv6 source address. The MR has to replace the IPv6 source address of the packets with its CoA and has to add a Home Address destination option carrying the LFN's address, and also recalculate the checksums (if needed).

The packet exchange of the proposed solution is depicted in Fig. 3. Initially, NEMO Basic Protocol [3] is used, as shown at the top of the figure, so packets between the $\mathrm{CN}$ and the LFN are encapsulated in a tunnel between the MR and its HA (HA_MR), following a suboptimal path. Triggered by this traffic flow, the MR starts the RR procedure for this LFN-CN pair. This is done by sending proper HoTI and CoTI messages to the CN. The MR processes the replies (HoT and CoT) messages from the $C N$. Once the RR procedure is completed, the MR is able to send a BU message to the $\mathrm{CN}$ on behalf of the LFN (i.e. using the LFN's address as Home Address). After that, the optimized communication flow is also shown -at the bottom of the figure-. Packets from the $\mathrm{CN}$ are sent to the MR's CoA, carrying a Type 2 Routing Header with Next Hop set to the LFN's address. The MR processes the packet, removing the Routing Header and recalculating packet checksums if needed. In the other sense -LFN to CNthe MR adds a Home Address destination option to the packets sent by the LFN and also replaces the IPv6 source address of the packets, setting this field to the MR's CoA. 


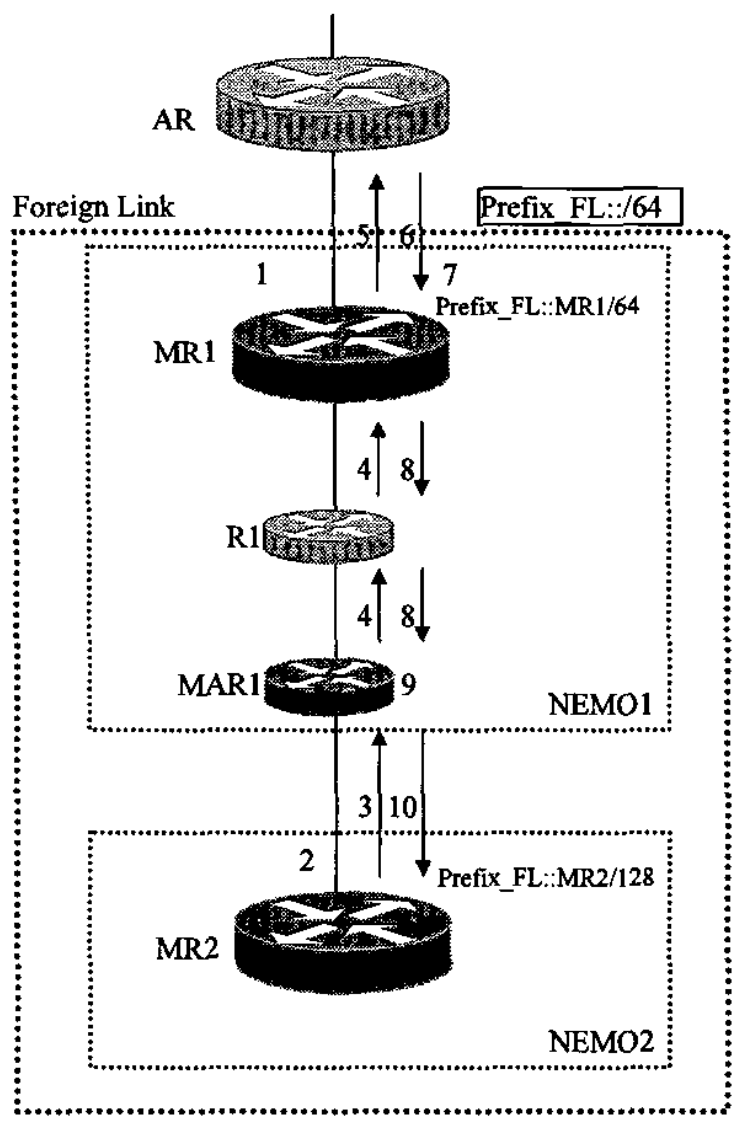

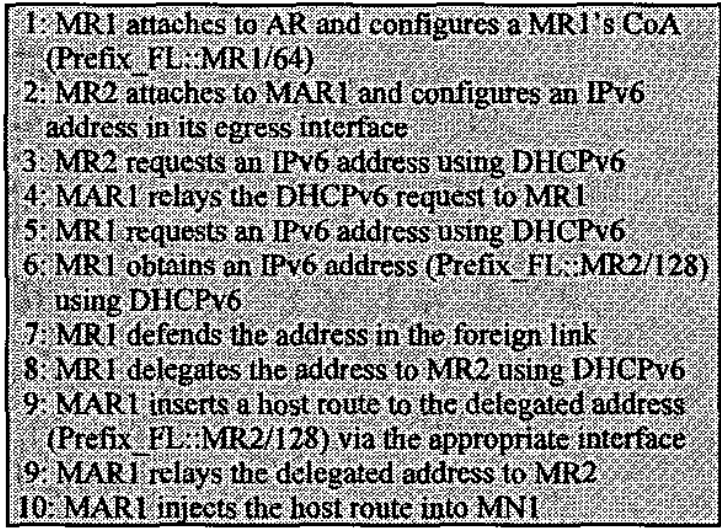

Fig. 4. Address Delegation mechanism with built-in capability

From the point of view of security, the fact of allowing the MR to perform some operations on behalf of the LFNs attached to it does not suppose a security threat, because the LFN trusts in its MR for the routing of all the traffic it sends/receives. Besides, the MR and the LFNs attached to it, belong to the same administrative domain, so no additional risks are introduced by the proposed mechanism.

\section{B. Nested Mobile Networks}

\section{B.1. Goals and requirements}

In the previous section we have described how the proposed MIRON mechanism works, allowing optimizing the route experimented by packets between a $\mathrm{CN}$ outside a single NEMO and a LFN inside. The goals of the solution for nested NEMOs are the same than in the single NEMO scenario, but extended to support an arbitrary number of nesting levels. As we will present in the next subsection, this will be done by deploying an address delegation mechanism with built-in routing capabilities.

\section{B.2. Proposed mechanism}

In order to extend the solution previously presented to support nested NEMOs, it is necessary to deploy some mechanism that provides topologically meaningful IPv6 addresses to the MRs in the nested mobile network hierarchy, so they can use them as MR's CoA in the Route Optimization procedure. Addresses that fit in this category are the ones belonging to the IPv6 space of the top Access Router (i.e. the Access Router that provides connectivity to top level MR -the root-MR- of the nested NEMO). Therefore, every Mobile Router within the nested NEMO should request and obtain an IPv6 address from the foreign network that the root-MR is visiting. Moreover, some routing capability should be also provided in order to enable the routing of packets to these delegated addresses inside the nested NEMO.

The operation of the address delegation mechanism with built-in routing capabilities will be described through an example (Fig. 4). A mobile network NEMO1 moves to a foreign link, and attaches to an access router AR, acquiring and configuring an IPv6 address (Prefix_FL::MR 1/64) as MRI's CoA on its egress interface. Mobile network NEMOI is formed by mobile router MR1, a non-NEMO and non- 
MIRON aware router R1 (fixed router) and, a MIRON Access Router (MAR) called MARl. A MAR is a modified access router that implements some operations required by MIRON solution (e.g. modified DHCPv6 [6] relaying function). This modification is needed in order to support mobile networks with fixed routers within the infrastructure (scenario not supported by many of the existing route optimization proposals). The MAR function can be also collocated in a MR in scenarios where the access router function is performed by a MR (i.e. there is no fixed routers within the NEMO).

When a second mobile network NEMO2 attaches to NEMO1, through MAR1, initially MR2 configures an IPv6 address in its egress interface belonging to the IPv6 addressing space of NEMO1, and then MR2 requests a topologically correct IPv6 address using DHCPv6 [6]. As it was explained above this address belongs to the IPv6 addressing space of the foreign link that it is visiting the rootMR, in this case MRl. Next, MAR1, acting as a DHCPv6 relay, forwards the request to MR1. Then, MR1 requests an IPv6 address in the foreign link using DHCPv6, obtains one (Prefix_FL::MR2/128) and starts to defend it on the foreign link. MR1 delegates the address to MR2 using the DHCPv6 relay router, MAR1. MAR1, when receiving the delegated address, first adds a host route to the delegated address in its routing table and very important, it injects this host route inside NEMO1 through an intra-domain routing protocol (only necessary if there is fixed routers within the NEMO). It should be noted that using host routes $(/ 128)$ does not suppose scalability issues, because intra-site routing systems can easily manage the required amount of routes generated by the expected number of sub-NEMO networks behind a rootNEMO.

Once this process is completed, MR2 has a topologically meaningful IPv6 address (belonging to the foreign link addressing space) that will be used as MR2's $\mathrm{CoA}$ by the MIRON mechanism. Packets from a CN to this MR2's CoA will reach $M R 2$ without any tunneling. A packet destined to MR2's CoA first arrives to the foreign link, where MR1 is defending this address. After getting this packet, MR1 looks up the routing table and uses the learned host route to forward the packet to the MAR1, which finally delivers it to the destination MR2.

The proposed mechanism of address delegation with built-in capabilities enables MIRON to provide route optimization also when nesting is involved. Every MR in the topology is able to perform the operations defined in the previous section, using a meaningful $\mathrm{CoA}$ obtained by means of the address delegation mechanism.

This solution provides Route Optimization support for nested and non-nested NEMOs. So, the solution provides RO support to the NEMO general solution.

\section{RELATED WORK}

In the last year, multiple solutions to provide partial route optimization support for NEMO have been presented. There are several proposals that try to solve this issue in different ways. Taxonomy of the different approaches to tackle the problem can be found in [7]. The proposal presented here fits in the category of solutions where the route optimization is performed between the MR and the CNs which MNN is communicating with. Provided that route optimization with correspondent nodes is initiated by the MR on behalf of LFNs, and as a result triangular routing through the $\mathrm{HA}$ is avoided.

In particular, most of the partial solutions are only focused on minimizing the number of tunnels required outside the NEMO when there are multiple levels of nesting. The most common approach in the literature is to have one bidirectional tunnel outside the NEMO, where the end-point of such a tunnel on the mobile side may be either the root-MR or the first MR on the egress path or in same cases, the own host if this is a Mobile Node (i.e. a node with MIPv6 functionalities). Usually the other end-point will be the respective Home Agent (i.e. the Home Agent of the root-MR, or of the first MR on the egress path, or of the Mobile Node). Two relevant proposals that follow this approach are [9], [10]. Both proposals have in common that avoid the multiple encapsulation of the traffic but maintain the home tunnel of the first MR on the egress path. In both cases the HA of this MR learns the ingress path towards this MR inside the nested NEMO and performs source routing using a routing header when sending packets through the corresponding tunnel. These approaches are different with regard to the way the HA leams the ingress path towards this MR inside the nested mobile network. In [9], a type of Record Route header, named Reverse Routing Header (RRH), is used. This RRH is appended by the first MR on the egress path when it is sending packets towards its HA through its reverse tunnel. Each MR on the egress path puts its CoA in the RRH. In this way, the $\mathrm{HA}$ receiving this packet can construct the chain of access routers the first MR on the egress path is attached to. It has been identified that this approach has security concerns. On the other hand, in [10] each MR on the egress path has to send an Binding Update towards its $\mathrm{HA}$ with the Access Router Option (ARO) including home-address of the access router it is currently attached to. This operation is much more complex but it also allows HA learning the ingress path to its MR.

Other approach to minimize the number of tunnels required outside the nested NEMO is that the root-MR, the sole MR with a topologically meaningful address, acts as a proxy in the MIPv6 registration [11]. In essence each MR sends the CoA of this root-MR to its $\mathrm{HA}$, so the HA maintain a tunnel with the root-MR. In addition the MR registers to root-MR and has to provide some information that allows the root-MR to find out the ingress path towards this MR inside the mobile network. For instance, the root-MR maintains a tunnel to each MR, a tunnel to the $\mathrm{HA}$ of each MR, and switches packets between the two.

Some authors have proposed asking for a prefix delegation [12] which enables that every node in the NEMO will have a topologically meaningful address. In this way, nodes outside the NEMO can send packets to a node inside 
the NEMO without adding a source routing header or using additional tunnels inside the mobile router. Nevertheless, in the approach presented in this paper when a MR is away from home and it is attached to a visited network it will ask to its access router for topologically meaningful hosts address and this $\mathrm{CoA}$ will be used by $\mathrm{CN}$ to send packets towards the LFN.

\section{Performance Evaluation}

In this section we will present some comparative results concerning the performance of the proposed solution in comparison with the NEMO Basic Support Protocol [3] and one of the proposals of route optimization when nesting is involved: Reverse Routing Header [9].

\section{A. Critical Parameters}

There are multiple parameters that can be used for comparison of different solutions. Nevertheless, when dealing with network mobility and the problem of the suboptimal routing introduced by the basic solution [3], some aspects are critical:

- Packet overhead. The overhead is important because it adds processing delay in intermediate hops and could reduce the Path MTU (PMTU).

- Number of HAs crossed by packets. In nested mobile networks, the pinball routing problem (i.e. packets going through all the MR's HAs) could appear when packets go through all (or some of them, if some optimization is employed) MRs' HAs. The MRs' HAs will, in general, not be in the path, so they are additional routing legs (i.e. partial routing paths that would not be needed if the route were optimal) added by the mobility basic support. Crossing HAs does not only mean additional hops but also adds packet delivery delays, because HAs could be far away each other.

\section{B. Packet Overhead comparison}

The overhead per packet introduced by the three approaches is the following:

- NEMO Basic Support Protocol [3] adds a tunnel (40 additional bytes) between the MR and its $\mathrm{HA}$ for each level of nesting. $L$ is the number of nesting levels (a 0 value means no nesting, i.e. a single NEMO)

$$
\text { PacketOverhead }=(L+1) * 40 \text { (bytes) }
$$

- Reverse Routing Header optimization [9] sets up a unique tunnel between the first MR on the egress path and its HA, but introduces a Reverse Routing Header (Type 4 Routing Header) in the LFN->CN direction and a multi-hop Type 2 Routing Header in the other direction.

$$
\text { PacketOverhead }=40+L * 16+8 \text { (bytes) }
$$

- MIRON does not add any tunnel. It only adds a Home Destination Address ( 24 bytes) or a Type 2 Routing Header ( 24 bytes), no matter how many nesting levels are involved.

$$
\text { PacketOverhead }=24 \text { (bytes) }
$$

The evolution of the packet overhead when increasing the levels of nesting (from 0-i.e. no nesting- to 6) is shown in Fig. 5. While packet overhead in both NEMO basic solution and $R R H$ approach grow with $L$, in MIRON remains constant at the lowest value ( 24 bytes).

\section{Number of HAs}

The number of HAs crossed in the three approaches is the following:

- NEMO Basic Support Protocol [3] adds a tunnel (1 crossed HA) between the MR and its HA for each level of nesting.

$$
\text { NumberOfHomeAgents }=\mathrm{L}+1
$$

- Reverse Routing Header optimization [9] sets up a unique tunnel between the Bottom Level Mobile Router and its HA. Therefore, there is only one intermediate hop:

$$
\text { NumberOfHomeAgents }=1
$$

- MIRON does not add any tunnel.

$$
\text { NumberOfHomeAgents }=0
$$

Taken into account the presented results, MIRON solution shows the best performance, both in terms of packet overhead and number of HAs.

\section{IMPLEMENTATION NOTES}

MIRON adds some changes in the operation of MR and HA from the one defined in [3]. Besides it adds the MIRON Access Router (MAR) role, that has to be present in every access router within the mobile network topology (this function may be collocated in the MR) in order to allow the presence of fixed routers within a NEMO. Next, we describe the most relevant parts of the implementation process:

- Major implementation concerns are related with the new MR functions. MRs must support the capability of performing the MIPv6 RO procedure on behalf of the LFNs. This can be done adapting an existing MN MIPv6 stack and using it in the MR. Key issues of this adaptation are the different operation of a $\mathrm{MN}$ 


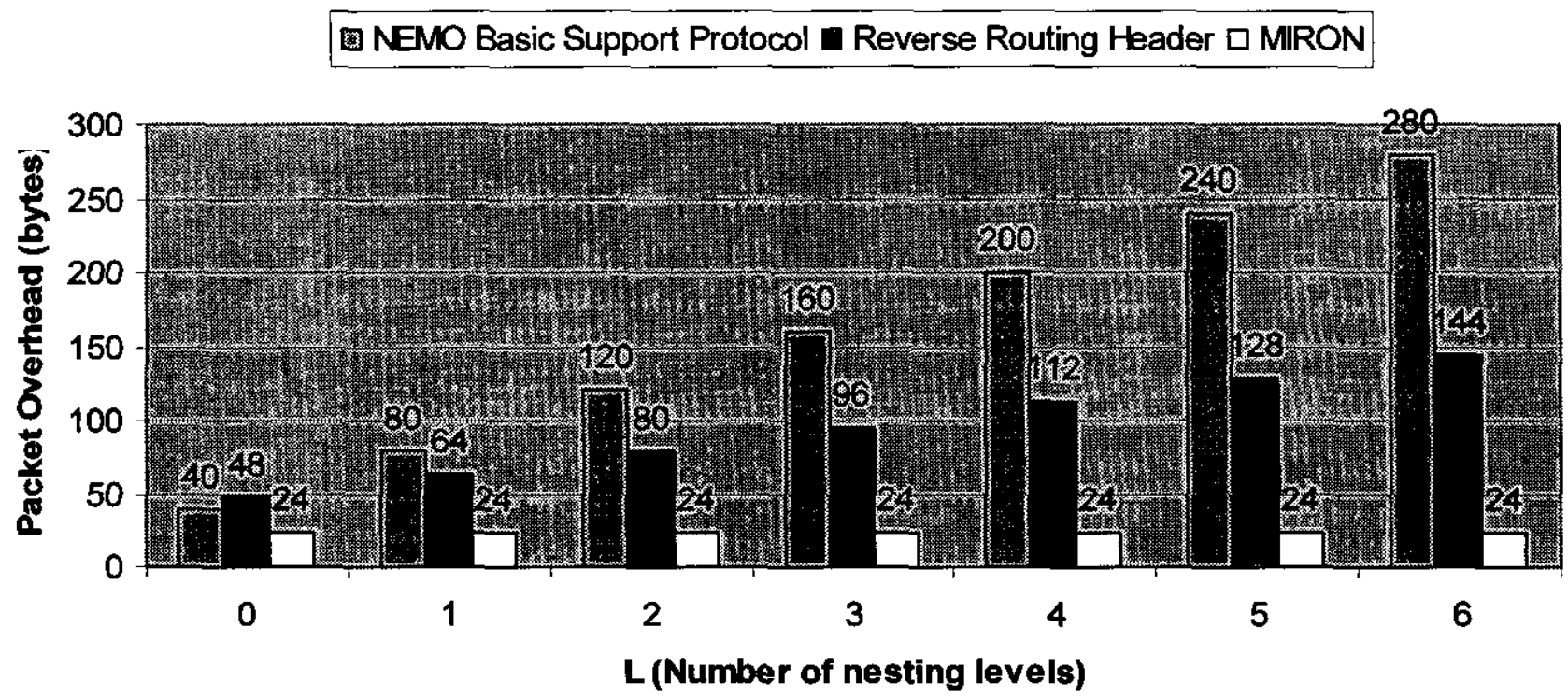

Fig. 5. Packet overhead vs. L (number of nesting levels)

and a router (i.e. mainly autoconfiguration procedures).

- Besides, the MRs must maintain new data structures that allow identifying which flows are being optimized and between which peers (CN and LFN). The MR should also maintain information related to the RR procedure and binding of each CN-LFN pair that is being route-optimized. All of this MIPv6related part of the implementation can be done by modifying and adding some functions to the existing MIPv6 implementation for Linux MIPL [14].

- A reduced set of DHCPv6 server capabilities must be added to the MR, in order to implement the recursive address request/delegation mechanism proposed. The access routers included in the mobile network (MARs) should be modified also, including a modified DHCPv6 relay operation. MARs must inject (if needed) host routes into the NEMO domain, using an intra-domain routing protocol. This part of the implementation could be done modifying the implementation of DHCPv6 for Linux [14], and the RIP or OSPF daemons provided by Quagga [15].

\section{CONCLUSIONS AND FUTURE WORK}

We have presented a route optimisation solution for mobile networks (NEMOs) based on Mobile IPv6, which allows the use of the route optimization support for MIPv6 available in $\mathrm{CN}$ to provide route optimisation for NEMOs. An advantage of this solution is that it would profit from the installed base of CNs that currently support MIPv6 toute optimization. Therefore, its adoption would be easier than if new $\mathrm{CN}$ mechanisms were required.
MIRON solution is valid in single mobile network scenarios and also in the new NEMO specific scenario of nested NEMOs. Some work have to be done in the identification, classification and study of the scenarios in which network mobility will play an important role, but some relevant ones are already very clear and likely to occur in the present and near future. For example, the scenario - already pointed in this paper - of public transportations (like trains or buses) that provide - while moving-Internet access to their travellers. Besides, the new NEMO specific scenario of nesting will be also very likely, for example, when PANs are introduced in the previous scenario. These are only two of the possible scenarios in which network mobility would be involved and that show that it is needed to deploy mechanisms to cope efficiently with the problem of the movement of networks. NEMO Basic Support Protocol [3] enables networks to move among different attachment points without interrupting the established communications of the nodes within, but presents some suboptimal routing problems, that add delays and packet overhead.

MIRON allows LFNs nodes within a NEMO to experience MIPv6 route optimization by enabling the MR to perform MIPv6 RO on behalf of them. A key advantage of the presented mechanism is that it does not change either $\mathrm{CN}$ or LFN operations. Besides, mobility remains being transparent to the LFNs (i.e. LFNs are not aware of network movement).

An address delegation mechanism with built-in routing capability has been proposed. This mechanism uses DHCPv6 protocol and adds some modifications to the DHCPv6 relay function. IPv6 addresses are recursively requested upstream using DHCPv6, until they reach the root-MR, which is actually who requests and obtains valid meaningful IPv6 addresses, that are then delegated back downstream. As the same time that these addresses are being delegated 
downstream by the MRs or MARs of each NEMO, host routes are injected in each of the mobile networks, in order to grant the global reachability of these addresses. This mechanism has no scalability or security concerns and enables the extension of the applicability of the route optimization mechanism to nested NEMOs, without adding any kind of performance penalty.

Performance quantitative results have been presented and show that the proposed mechanism introduces minimal packet overhead ( 24 bytes per packet) that, in addition, remains constant despite the number of nested NEMOs. In the other hand, the packet overhead added by the NEMO basic solution grows fast with the number of nested mobile networks. The Reverse Routing Header ( $\mathrm{RRH}$ ) proposal also adds significant packet overhead, that grows too when more nested mobile networks are present. An additional parameter has been studied: the number of HAs that are present in the communication path between a LFN and a CN. This number represents additional hops that would not be necessary if an optimal routing is achieved, and that, in general, add packet delivery delays. Again, NEMO basic solution obtains the worse results, adding one HA per level of nesting. RRH only adds a HA in the path for any number of levels of nesting (this is the major advantage of that proposal), but MIRON solution does not add any HA in the communication path, due to the application of the proposed address delegation mechanism with built-in capabilities.

Further work remains to be done. The extension of the applicability of this solution also to Visiting Mobile Nodes (VMNs) and Local Mobile Nodes (LMNs) [4] should be studied. Another point, already mentioned, is the study of usage scenarios of network mobility in order to identify which situations are more important and likely to occur and what speciftc problems are present in these scenarios. In this way, route optimization solutions for these 'key situations' could be provided. Other important issue is the design of effective triggering mechanisms for the activation of the route optimization procedure (i.e. the decision to activate or not the MIRON procedure for a communication flow between a LFN and a $\mathrm{CN}$ ).

\section{ACENOWLEDGEMENTS}

The authors would like to thank Prof. Ignacio Soto for his revision of the paper and advice on this topic.

\section{REFERENCES}

[1] C. Perkins "IP Mobility Support for IPv4", RFC 3344. August 2002

[2] D. Johnson "Mobility Support in IPv6", draft-ietfmobileip-ipv6-24, work-in-progress, June 2003

[3] Vijay Devarapalli et al. "Network Mobility (NEMO) Basic Support Protocol", draft-ietf-nemo-basic-support02, work-in-progress, December 2003

[4] T. Emst et al. "Network Mobility Support Terminology", draft-ietf-nemo-terminology-01, work-in-progress, February 2004

[5] T. Emst "Network Mobility Support Goals and Requirements", draft-ietf-nemo-requirements-02, workin-progress, February 2004

[6] R. Droms et al. "Dynamic Host Configuration Protocol for IPv6 (DHCPv6)", RFC 3315, July 2003

[7] P. Thubert et al. "Taxonomy of Route Optimization models in the Nemo Context", draft-thubert-nemo-rotaxonomy-02.txt, work-in-progress, February 2004

[8] H. Ohnishi et al. "HMIP based Route optimization method in a mobile network", draft-ohnishi-nemo-rohmip-00, work-in-progress, October 2003

[9] P. Thubert et al, "IPv6 Reverse Routing Header and its application to Mobile Networks", draft-thubert-nemoreverse-routing-header-04, work-in-progress, February 2004

[10] C-W Ng et al. "Securing Nested Tunnels Optimization with Access Router Option", draft-ng-nemo-accessrouter-option-00, October 2002

[11]H. Kang et al. "Route Optimization for Mobile Network by Using Bi-directional Between Home Agent and Top Level Mobile Router", Work in progress, Draft-hkangnemo-ro-tlmr-00.txt.December 2003.

[12] K-J Lee et al. "Route Optimization for Mobiles Nodes in Mobile Network based on Prefix Delegation", draftleekj-nemo-ro-pd-02, work-in-progress, February 2004

[13] Mobile IPv6 for Linux (MIPL), http://www.mobileipv6.org

[14]DHCPv6 for Linux, http://hcpv6.sourceforge.net/

[15]Quagga Routing Suite, http:/www.quagga.net/

[16] DAIDALOS, http://www.ist-daidalos.org/ 